\title{
Endoscopic dacryocystrhinostomy
}

\section{Bernardo Cunha Araujo Filho ${ }^{1}$, Richard Louis Voegels $^{2}$, Ossamu Butugan ${ }^{3}$, Carlos Diogenes Pinheiro Neto $^{4}$, Marcus Miranda Lessa ${ }^{5}$}

Key words: dacryocystrhinostomy, endoscopic, endonasal, dacryocystitis.

\section{Summary}

\section{F} and endoscopic dacryocystorhinostomy (EN-DCR) is now a well-established procedure to relieve nasolacrimal duct obstruction, becoming its domain for the ENT surgeons indispensable. Aim: The aim of the present study is to report the experience of the Otorhinolaryngology Department of the University of São Paulo Medical School in the management of the obstruction of the drainage of the nasolacrimal system by EN-DCR, comparing with the results in literature. Study design: clinical retrospective. Material and Method: We reviewed the medical records of 17 patients (17 eyes) that were submitted to EN-DCR between april 2001 and july 2004. We analysed: sex, age at the time of diagnosis, etiology, clinical findings, surgical technique, use of silicone tubes, follow-up and complications. Results: Eight men and nine women, the age range was from 29 to 79 years (mean 42.6413.1 years), mean follow-up time: 15 months, presented a lacrimal clinic with epiphora. Powered DCR was performed in 06 cases and YAG LASER in 01 patient. Silicone tubes were used in all cases and left in place mean 7.9 weeks. The surgical success rate was $82,3 \%$. Conclusion: EN-DCR showed one safe technique, with advantages in relation to the external technique. So ophthalmologists and ENT physicians must work in harmony to offer more benefits to its patients.

${ }^{1}$ Ph.D. studies under course, Division of Clinical Otorhinolaryngology, HCFMUSP, Specialist in Otorhinolaryngology, SBORL

${ }^{2}$ Associate Professor, Discipline of Otorhinolaryngology, Medical School, University of Sao Paulo, Director of Rhinology, HCFMUSP. ${ }^{3}$ Full Professor, Discipline of Otorhinolaryngology, Medical School, University of Sao Paulo.

${ }^{4}$ Resident Physician, Division of Clinical Otorhinolaryngology, Hospital das Clínicas, Medical School, University of Sao Paulo.

${ }^{5}$ Ph.D. in Otorhinolaryngology, HCFMUSP, Professor of Otorhinolaryngology, Faculdade Federal da Bahia.

Division of Clinical Otorhinolaryngology, Hospital das Clínicas, Medical School, University of Sao Paulo.

Address correspondence to: Bernardo Cunha Araújo Filho - Rua Oscar Freire 171 apt. 1101 05409-011 Sao Paulo SP. Tel (55 11) 8319-4444 - E-mail: bcaf@terra.com.br

The present article was submitted through SGP on June 15, 2005 and approved on September 8, 2005. 


\section{INTRODUCTION}

Infection of the lachrymal pathways has attracted the attention of physicians for some time, however, as a result of the development of antibiotics, acute forms do not represent a life-threatening condition as they used to in the past, and at the same time the chronic forms became more prevalent.

The history of lacrimal pathway surgery dates back from Hamurabi $(2,200 \text { B.C. })^{1}$. Since then, techniques for treatment of lacrimal pathways have been developed, fighting against infections and restoring the transit of tears through the lacrimal system. Thus, dacryocystorhinostomy (DCR) has been the treatment of choice for cases of distal obstruction of lacrimal system (below the common canaliculi) and consists in creating an anastomosis between the lacrimal sac and the basal cavity, enabling the tear to drain to the lower meatus, relieving the symptoms.

Technological innovations and surgeries of lacrimal system that are less invasive were developed to reduce morbidity and to improve the results. Traditionally, the treatment of nasolacrimal obstruction is external DCR, frequently performed by ophthalmologists. This technique was described by Toti in 1904 and modified by Dupuy-Dutemps and Bourguet ${ }^{2}$, with the suture of mucous flaps.

The endonasal approach was described for the first time by Caldwell in $1893^{3}$, but it was forgotten for decades by the limited vision and the assessment of nasosinusal anatomy. The introduction of microscope and later the endoscopic techniques, associated with the close relation of lacrimal systems and the nasal fossa, have made endonasal surgical treatment of low lacrimal affections very popular among Otorhinolaryngologists ${ }^{4}$. Currently, endoscopic DCR is a well-accepted and established technique in the treatment of obstruction of the lacrimal sac and nasolacrimal duct ${ }^{1,3,5}$ and its proficiency by Otorhinolaryngologists has become indispensable.

The purpose of the present article is to present the experience in endoscopic DCR of the Division of Clinical Otorhinolaryngology, HCFMUSP, comparing and discussing our results with those found in the literature.

\section{MATERIAL AND METHODS}

This study was based on the retrospective analysis of 17 medical charts of patients submitted to endoscopic DCR, admitted to the Division of Clinical Otorhinolaryngology, Hospital das Clínicas, Medical School, University of Sao Paulo, in the period between April 2001 and July 2004. Out of 17 patients (17 eyes), 8 were men (47.1\%) and 9 were women (52.9\%). The age at diagnosis ranged from 29 to 79 years, with mean age of 42.64 years and standard deviation of 13.1 years.

Patients were assessed according to: a. Gender

b. Age at time of surgery

c. Etiology

d. Clinical presentation

e. Surgical technique performed (cold or laser instrument)

f. Use of silicone tube and duration of insertion

g. Number of surgeries necessary to correct

h. Some aspects of postoperative follow-up, such as the use of antibiotics and the observed complications.

The results were statistically analyzed with Fischer exact test and significance level of $\mathrm{p}<0.05$.

\section{Surgical technique}

Procedures were performed under general anesthesia, but in one case (LASER YAG) we used local anesthesia with sedation. We preferred general anesthesia owing to greater comfort of the patients and possible correction of septal deviations, bullous and paradoxical conchae, if necessary. After topical vasoconstriction of nasal cavity with lidocaine solution at 2\% with adrenaline 1:2,000, we infiltrated lidocaine $2 \%$ with adrenaline at 1:80,000 in the anterior region to the medium concha. We used 0 degree and $4 \mathrm{~mm}$ endoscopes (Hopkins-Karl Storz). Under endoscopic visualization, we made a mucous rectangular flap with the posterior base adjacent to the medium concha, 1 cm2 subperiosteal using Sickle Knife and detacher-aspirator or Cottle. We positioned the flap posteriorly during the procedure, protecting the anterior insertion of the medium concha against traumas. After exposure of lacrimal bone and frontal process of maxilla, we created a posterior window to access the sac and expanded the window anteriorly to expose the whole width of the lacrimal sac. In 6 patients we used power-driven instruments (motor and diamond bur) and in one case we used LASER YAG. We also used cold instruments (such as chisel and hammer) to prepare a rhinostome.

The lower limit of the bone window was the insertion of the lower concha on the nasal wall. The identification of the sac was made with a probe (Bowman) and through the canaliculi, it penetrated into the sac and was pushed medially, but it could also in some occasions be visualized by digital compression of the ocular medial cantus. It is advocated to remove the whole medial wall of nasolacrimal sac with clamping or Sickle Knife. Finally we cut a U-shaped mucous flap with anterior concavity to promote first intention healing, preventing bone exposure and less formation of granulomas.

A silicone tube was passed through the superior and inferior canaliculi up to nasal fossa and fixed in the vestibule, using nylon thread 3-0, maintaining the opening of the lacrimal pathways with the nasal fossa. We did not use any type of nasal packing. Frequent irrigation 
of the canaliculi with sterile solution was performed at outpatient level.

\section{RESULTS}

Epiphora was the predominant complaint in all cases. The main complaint of lacrimal obstruction was idiopathic (58.8\%), followed by septal surgeries and nasal conchae (17\%) and trauma (11.2\%). Synechia caused by transphenoid access for exeresis of macroadenoma of hypophysis and paranasal sinuses functional surgery were responsible for 2 cases. The mean time of follow-up was 15 months (minimum: 5 months and maximum: 37 months) and in all of the cases, we used silicone tubes for an average period of 7.9 weeks.

Four patients had been submitted to external DCR and were submitted to endoscopic procedure by obstruction of rhinostome. In one of these cases, septal deviation was responsible for the failure. Considering as surgical success the resolution of epiphora, the success of the endonasal technique was achieved in $82.3 \%$ of the cases. We observed that in one of the failures, we had employed LASER YAG. All recurrences have progressed well after the new endoscopic procedure up to the present moment.

Systemic antibiotics, such as first generation cephalosporins and topical ophthalmic drugs were used in the postoperative period, as well as frequent nasal lavage with sterile solution.

\section{DISCUSSION}

In this study, we analyzed 17 patients who presented epiphora in a period of 3 years. The largest series found in the literature was described by Sprekelsen et al.6 in 1996, with 152 eyes submitted to DCR, followed by Yang et al. ${ }^{7}$. However, the criteria for therapeutic success and the postoperative follow-up are varied, hindering the comparison of results among the many different authors.

The stenosis of lacrimal paths, idiopathic in two thirds of the cases 1 , family predisposition, anatomical variations of the duct and recurrent infection have been considered as possible causes. Our sample presented 58.2\% of the cases caused by idiopathic stenosis, presenting a value close to the one described in the literature. Similarly to what was observed in our study, chronic sinusitis of maxillary and ethmoidal sinuses, septal deviations and acute rhinitis may cause ascending infection of the duct, resulting in inflammatory reaction, edema, ulceration and finally, chronic dacryocystitis. Bilateral stenosis is $\operatorname{rare}^{1,8}$, as observed by us.

Only one case was treated with LASER and the result was poor. The endoscopic approach with LASER was proposed to improving hemostasis in the endoscopic surgery and to reduce the formation of granulation tissue. However, despite these advantages, its high price and low effectiveness ( $64 \%$ to $85 \%$ ), especially owing to the small ostium produced, has limited use to the technique i, $^{1,10}$

Cokkeser et al. ${ }^{3}$ advocated the use of chisel and hammer because it is a simpler, more hemostatic, cheaper and practical method to make a bone window. Conversely, Wormald et al. ${ }^{11}$, Sham et al. ${ }^{12}$ and Hartikainen et al. ${ }^{2}$ used drills to cause saccular exposure and ensure that through these instruments there is exposure of the more reliable lacrimal sac, which is ensured by the use of the chisel. Using the endonasal technique with chisel and hammer, and the use of power-driven drills, we did not observe any significant difference between these two approaches ( $\mathrm{p} \leq 0.05$ ). Our results were similar to the findings in the literature.

We observed that endonasal access provided easy access to repair affections that could impair the results, such as septal deviations, bullous concha, synechia, such as observed in patients submitted to previous external DCR, a very flexible method to endonasal anatomical affections.

Regardless of the methods used, it is known that, despite the controversy, a wide bone window and exposure of the whole sac is determinant in surgical success. Cokkerser et al. ${ }^{3}$, Sham et al. ${ }^{12}$, Wormald et al. ${ }^{13}$ consider that the removal of the whole bone from the sac is associated with better index of success and visualization of opening of common canaliculi, after the removal of the media wall of the sac, which is a good indicator of appropriate saccular exposure ${ }^{11}$.

Lindberg et al. ${ }^{14}$, in their study, observed that the final size of rhinostome did not have any relation with the size of the bone window, however Heher et al. ${ }^{15}$, based on this assumption, managed to have a rate of only $70 \%$ success. Confirming the reported data, Welham et al. ${ }^{16}$, reviewed 205 failed DCR and concluded that the main cause of failure was incomplete opening of rhinostome and Millman et al. ${ }^{17}$ observed that fibrotic sacs presented therapeutic failure in $29 \%$, whereas sacs with mucoceles had $82 \%$ failure.

At the end of the surgical procedure we may apply mitomycin C, preventing proliferation of scarring tissue $e^{1,18,19}$ and also mucous flap sutures. In our sample, we did not use these methods, but the use of silicone stents to prevent stenosis of rhinostome was used in all patients. Silicone tubes are used to maintain the opening of the ostium, and normally they are placed for a varied period of time, according to the authors' preference. Similarly to our study, Kong et al. ${ }^{10}$ advocated a maximum of 8 weeks. Unlu et al. ${ }^{20}$, comparing the groups with and without intubation with silicone after endoscopic DCR did not find significant results between the two groups ( $92 \%$ versus $87 \%$, respectively). However, the level of complications was greater in the group of patients with intubation, despite the fact that Walland et al. ${ }^{21}$ reported that the use of silicone tubes did not increase the risk of stenosis. 
Broad-spectrum systemic antibiotics and topical ophthalmologic drugs are used postoperatively. Similarly to the use of antibiotics, frequent daily nasal lavage with sterile solution and irrigation of lacrimal paths are essential in therapeutic success.

The DCR EN results ranged from $70 \%$ to $95 \%$ and depend on a series of factors, already reported. Cokkeser et al. ${ }^{3}$, comparing DCR EX to DCR EN in 115 patients had success rates of $89.8 \%$ and $88.2 \%$, respectively, and recommend the use of endonasal techniques owing to their advantages comparing to the external approach. The success of the endonasal technique in our service was $82.3 \%$, similarly to the results reported in the literature.

Many studies reported similar results or slight nonsignificant disadvantage of DCR EN in comparison to DCR EX. Hartikainem et al. ${ }^{2}$ compared these two techniques in 64 cases and found success rates in 75\% for DCR EN and 91\% for DCR EX. These poor results may be explained by the fact that these patients have been submitted to small rhinostomies, with $7 \mathrm{~mm}$ diameter, emphasizing the importance of a wide window between the sac and the nasal cavity.

The main complication is therapeutic failure 16. In three cases, we did not succeed in the first endonasal approach, but all recurrences progressed well after the new endoscopic procedure. The reasons for failure may be owed to many different factors: excessive bleeding, wrong location of lacrimal sac, incision of inappropriate mucosa (leading to synechia and excessive granulation), failure in bone opening, bone neoformation, canalicular obstruction, SUMP syndrome and affections inherent to the nasal fossa (narrow nasal fossa, mucocele, etc). Other complications are: soft tissue infection after surgery (8\%), bleeding, orbital complications, such as fat prolapse, corneal irritation, and nasal discomfort caused by silicone tubes ${ }^{1,4,10,19}$. Christmas et al. ${ }^{22}$ reported the complication of DCR EM that, owing to formation of synechia, caused blocked drainage of middle meatus, leading to frontal-ethmoidal sinusitis, a fact that should warn the Otorhinolaryngologists about the possible complications inherent to the procedure.

\section{CONCLUSION}

Endoscopic technique is a safe technique with promising results that provide a series of advantages compared to the external technique, such as avoiding scarring of the face and disarrangement of the medial canthal anatomy.

The two techniques, external and endoscopic, in experienced hands, present similar results and ophthalmologists and otorhinolaryngologists should work in harmony to provide more benefits to their patients.

Table 1. Review of the literature about endonasal dacryocystorhinostomy.

\begin{tabular}{|c|c|c|c|c|c|}
\hline Authors & Year & $\begin{array}{c}\text { Number of } \\
\text { eyes }\end{array}$ & $\begin{array}{l}\text { Rate of } \\
\text { success }\end{array}$ & Follow up & Comments \\
\hline Sprekelsen et al. ${ }^{6}$ & 1996 & 152 & $96 \%$ & 12 months & \\
\hline Yang et al. ${ }^{7}$ & 1998 & 150 & $90 \%$ & 13 months & $\begin{array}{l}\text { Used uncinate process as the main } \\
\text { repair sac }\end{array}$ \\
\hline Hartikainem et al. ${ }^{2}$ & 1998 & 32 & $75 \%$ & 12 months & $\begin{array}{l}\text { Rhinostomy presented maximum } \\
\text { diameter of } \\
\text { 7mm (facilitating re-obstruction) }\end{array}$ \\
\hline Sham et al. ${ }^{12}$ & 2000 & 17 & $88 \%$ & 13 months & $\begin{array}{l}\text { Failure caused by orbital fat prolapse } \\
\text { and canaliculi obstruction }\end{array}$ \\
\hline Cokkeser et al. ${ }^{3}$ & 2000 & 51 & $88,2 \%$ & 25 months & $\begin{array}{l}\text { Used silicone tube for } 2 \text { to } 3 \\
\text { months. } \\
\text { Failure by learning curve in }\end{array}$ \\
\hline HCFMUSP & 2004 & 17 & $82,3 \%$ & 15 months & $\begin{array}{l}\text { Used silicone tube on average for } \\
7.9 \text { weeks. }\end{array}$ \\
\hline
\end{tabular}




\section{REFERENCES}

1. Onerci M. Dacryocystorhinostomy. Diagnosis and treatment of nasolacrimal canal obstructions. Rhinology 2002; 40: 49-65.

2. Hartikainen J, Antila J, Varpula M, Puukka P, Grenman R. Prospective randomized comparison of endonasal endoscopic dacryocystorhinostomy and external dacryocystorhinostomy. Laryngoscope 1998; 108: 1861-6.

3. Cokkeser Y, Er H. Comparative external versus endoscopic dacryocystorhinostomy. Otolaryngol Head and Neck Surg 2000; 123: 488-91.

4. Cunningham MJ, Woog JJ. Endonasal endoscopic dacryocystorhinostomy. Arch Otolaryngol Head and Neck Surg 1998; 124: 328-33.

5. Bartley GB, Abou-Rayyah Y, Rose GE. Pediatrics dacryocystorhinostomy for nasolacrimal duct obstruction. Ophthalmology 2000; 108: 1562-4.

6. Sprekelsen MB, Barberan MT. Endoscopic Dacryocystorhinostomy: Surgical Technique and results. Laryngoscope 1996; 106: 187-9.

7. Yang MW, Hardman-Lea S. Endoscopic inferior dacryocystorhinostomy. Clin Otolaryngol 1998; 23: 152-7.

8. Tos M, Balle V, Andersen R. Dacryocystorhinostomy. Ann Otol Rhinol Laryngol 1986; 95: 352-5.

9. Metson R, Woog JJ, Puliafito CA. Endoscopic laser dacryocystorhinostomy. Laryngoscope 1994; 104: 269-74.

10. Kong YT, Kim TI, Byung WK. A report of 131 cases of endoscopic laser lacrimal surgery. Ophthalmology 1994; 101: 1793-800.

11. Wormald PJ. Powered Endoscopic Dacryocystorhinostomy. Laryngoscope 2002; 112: 69-72.

12. Sham CL, Hasselt AV. Endoscopic terminal dacryocystorhinostomy. Laryngoscope 2000; 110: 1045-9.
13. Wormald PJ, Kew J, Hasselt AV. Intranasal anatomy of the nasolacrimal sac in endoscopic dacryocystorhinostomy. Otolaryngology - Head and Neck Surgery 2000; 123: 307-10.

14. Lindberg JV, Anderson RL, Bumsted RM. Study of intranasal ostium in external dacryocystorhinostomy. Arch Ophthalmol 1982; 100: 1758-62.

15. Hehar SS, Jones NS, Sadiq A. Endoscopic Holmium: YAG-Laser Dacryocystorhinostomy- safe and effective as a day-case procedure. J Laryngol Otol 1997; 111: 1056-9.

16. Welham RA, Wulc AE. Management of Unsuccessful lacrimal surgery. Br J Ophthalmol 1987; 71: 152-7.

17. Mannor G, Millman A. The prognostic value of preoperative dacryocystography in endoscopic intranasal dacryocystorhinostomy. Am J Ophthamol 1992; 113: 134-7.

18. Marks SC. Nasal And Sinus Surgery: Philadelphia: W.B. Saunders Company; 2000.

19. Hauslaer R, Caversaccio M. Microsurgical endonasal dacryocystorhinostomy with long term insertion of bicanalicular silicone tubes. Arch Otolaryngol Head and Neck Surg 1998; 124: 188-91.

20. Unlu HH, Aslan A, Toprak B, Guler C. Comparison of Surgical outcomes in primary endoscopic dacryocystorhinostomy with and without silicone intubations. Annals Otol Rhinol Laryngol 2002; 111: 704-9.

21. Walland MJ, Rose GE. The effect of silicone intubation on failure and infection rates after dacryocystorhinostomy. Ophthamol Surgery 1994; 25: 597-600.

22. Christmas D, Mirante JP, Yanagisawa E. Middle meatal obstruction following endoscopic dacryocystorhinostomy. Ear Nose and Throat 2002; 81 (7): 431-2.

23. Minasian M, Olver JM. The value of nasal endoscopy after dacryocystorhinostomy. Orbit 1999; 18 (3): 167-76. 